\title{
Prix Luc Ciompi 2017
}

Kaspar Aebi

Dr, Président au Congrès annuel de la Société Suisse de psychiatrie et de psychothérapie

\author{
Lors de son Congrès annuel, la Société Suisse de psychiatrie et de psychothérapie \\ (SSPP) a décerné le prix Luc Ciompi à la Doctoresse Gemma Modinos. Ce prix donné \\ par le psychiatre et auteur Luc Ciompi est destiné aux travaux scientifiques de \\ valeur portant sur les interactions entre émotion et cognition et leurs consé- \\ quences pour la compréhension et le traitement de la schizophrénie.
}

Luc Ciompi n'est pas uniquement le fondateur du concept de la logique affective, l'enseignement des interactions entre pensée et sentiment, il a également contribué au développement du modèle vulnérabilitéstress qui permet de mieux comprendre les causes multisectorielles et les évolutions de la schizophrénie. Agé aujourd'hui de 88 ans, le chercheur a travaillé de 1977 à 1994 comme professeur de psychiatrie et directeur médical à la clinique de psychiatrie sociale de l'université de Berne. Durant cette période, il fonda également le foyer Soteria à Berne, dans le cadre duquel des personnes atteintes de troubles psychotiques aigus sont primairement traitées par un accompagnement et une aide sociale dans un environnement détendu. Les presque soixante ans d'activité de Luc Ciompi sont marqués par une compréhension psycho-socio-biologique intégrative des troubles psychiques. Sa curiosité scientifique a poussé le pionnier de la psychiatrie intégrative à créer un prix pour les travaux scientifiques de valeur portant sur les interactions entre émotion et cognition et leurs conséquences pour la compréhension et le traitement moderne de la schizophrénie. "Décerné pour la seconde fois en 2017, le prix est attribué à des travaux contribuant à confirmer ou infirmer la compréhension de la psychose de la logique affective», dit le fondateur Luc Ciompi.

\section{Recherches sur les facteurs déclenchants sous-jacents de la schizophrénie}

Les travaux de la lauréate du prix Luc Ciompi 2017 portent précisément sur cette approche: les études de la Doctoresse Gemma Modinos permettent de mieux comprendre les mécanismes neuronaux des réactions émotionnelles et de stress aux stades précoces de la schizophrénie. «Ma passion scientifique va à cette interaction entre ce que ressentent les personnes, ce qu'elles pensent et comment elles agissent ensuite», dit la chercheuse lors de la remise du prix. Ses projets se basent sur une combinaison de procédures d'imagerie et d'expériences comportementales et psychophysiques. Elle souhaite ainsi comprendre comment intervenir préventivement ou thérapeutiquement dans ces mécanismes pour empêcher ou retarder la psychose. Modinos s'est depuis toujours intéressée à «la question comment le cerveau peut créer le phénomène des symptômes psychotiques». Elle a obtenu un Bachelor of Science en psychologie ainsi qu'un Master of Science en neurosciences appliquées et un doctorat en neurosciences. Depuis 2010 elle travaille sur la neurobiologie de la psychose à l'institut de psychiatrie, psychologie et neurosciences du King's College de Londres. La chercheuse et professeure de 36 ans a publié plus de 30 ouvrages sur la neuropsychologie et a déjà reçu de nombreux autres prix de recherche. Ses travaux soumis pour le prix Luc Ciompi sont remarquablement différenciés et affinés quant à leur méthodologie. Elle démontre que les personnes de plus en plus vulnérables à la schizophrénie selon des critères cliniques objectifs et neurobiologiques, se caractérisent par une hyperréactivité émotionnelle et une activation excessive liée de certaines structures cérébrales cortico-limbiques. Elle a par ailleurs établi des corrélations intéressantes, mais parfois contradictoires avec le métabolisme du glutamate - un neurotransmetteur. «Mon objectif est d'identifier un sousgroupe qui pourra ensuite bénéficier d'un traitement précoce», dit Gemma Modinos. Selon le jury du prix, ses recherches fondent trois thèses fondamentales de la logique affective: les interactions étroites permanentes entre émotion et cognition; l'importance centrale des émotions dans la schizophrénie et les implications de ce résultat pour la genèse, la détection précoce et la prophylaxie de la maladie. 